\title{
A Pesquisa em tomada de Decisão Estratégica no Alto ESCALÃO: EVOLUÇÃo E bASE INTELECTUAL do temA
}

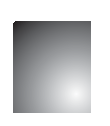

\author{
The Research in Top Management Team Strategic Decision- \\ Making: evolution and intelectual basis
}

\begin{abstract}
Bernardo Paraiso de Campos Serra
Mestre em administração pela Pontifícia Universidade Católica do Rio de Janeiro. Departamento de Psicologia. Rio de Janeiro, RJ. Brasil. E-mail: bernardoserra@outlook.com
\end{abstract}

\section{Patrícia Amélia Tomei}

Doutora em administração e Professora da Pontifícia Universidade Católica do Rio de Janeiro IAG. Rio de Janeiro, RJ. Brasil.

E-mail: patomei@iag.puc.rio

Fernando António Ribeiro Serra

Doutor em Materiais e Professor e Pesquisador do Programa de Pós-Gradução em administração. Universidade Nove de Julho -

UNINOVE. São Paulo, SP. Brasil. E-mail: fernandoars@uninove.br

\section{Resumo}

Este artigo tem como objetivo compreender a estrutura intelectual que conecta teorias a autores que pesquisam a tomada de decisão estratégica do alto escalão $e$ propor uma agenda para pesquisa futura no tema. Utilizou-se para este fim de pesquisa bibliométrica de citação e cocitação dos artigos publicados em periódicos internacionais sobre tomada de decisão, identificando os trabalhos mais referenciados, as publicações mais relevantes e mostrando as relações conceituais entre os trabalhos que fundamentam o tema $e$ identificam tendências dos estudos sobre o tema. Foram identificados três clusters teóricos: as características do Top Management Team (TMT) e como elas influenciam a tomada de decisão; fatores ambientais e sua influência na tomada de decisão, juntamente com a exposição de modelos e processos de tomada de decisão; conflito e consenso na tomada de decisão. A discussão permitiu ilustrar o atual estado do tema e suas lacunas e apresentar sugestões para pesquisas futuras.

Palavras-chave: Tomada de Decisão. Alto Escalão. Time de Gestão de Topo. Estratégia. Bibliometria.

\section{Abstract}

This article aims to understand the intellectual structure that connects theories and authors that research strategic decision-making on upper echelons and propose a research agenda for future research on strategic decision-making. We used a bibliometric research of articles published in international journals. A bibliometric analysis of citation and cocitation was conducted on the content studied in the articles of decision making, identifying the most referenced works and the conceptual relations between the work underlying the theme and trends. We identified three theoretical clusters on the subject: the characteristics of the Top Management Team (TMT) and how they influence decision making; how environmental factors compromise the decision making, along with the explanatory models and decision-making processes; conflict and allowed to present the current state of the subject and its gaps, and suggestions for future research.

Keywords: Decision-making. Upper Echelons. Top Management Team. Strategy. Bibliometry. 


\section{INTRODUÇÃo}

A gestão de topo das empresas pode ser um dos recursos estratégicos fundamentais. Apesar da falta de consenso em relação à abordagem e a como identificar quais recursos proporcionam uma efetiva vantagem competitiva. Hoskisson et al. (1999) e Serra e Ferreira (2010) propõem a consideração dos CEOs e equipes de gestão de topo como recurso estratégico. Hoskinson et al. (1999) inclusive apontam que os gestores de topo, representados pela Teoria do Alto Escalão de Hambrick e Mason (1984) são um subcampo da VBR. O artigo de Hambrick e Mason (1984) é considerado o estudo inaugural sobre a importância e características de gestão de topo, apresentando uma proposta de agenda de pesquisa sobre o papel dos gestores de topo e sua influência na estratégia e desempenho das organizações (PAPADAKIS; BARWISE, 2002). A importância do papel dos gestores de topo e do CEO está relacionada à influência destes no processo de tomada de decisão estratégica (RAJAGOPALAN; RASHEED; DATTA, 1993), especificamente em como as decisões influenciam as organizações. Apesar da importância do tema, e dos trabalhos de autores como Donald Hambrick sobre o papel dos CEOs e sua influência nas empresas (CANNELLA JR., 2001), ainda é preciso compreender melhor o $\mathrm{CEO}$ e os gestores de topo em relação à tomada de decisão estratégica. (SERRA; FERREIRA, 2010)

Apesar das diversas teorias e abordagens relacionadas à estratégia, a tomada de decisão estratégica pelo $\mathrm{CEO}$, só, ou em conjunto com a equipe de gestores de topo, aparenta ser crucial na estratégia. Papadakis e Barwise (2002) e Serra e Ferreira (2010), autores dedicados a estudar o papel do alto escalão dentro das organizações, abordam que entre os principais temas estudados em estratégia estão o papel dos gestores de topo. Mesmo Papadakis e Barwise (2002) já argumentaram que dois dos principais temas de estudo recentes em estratégia são o papel da administração de topo (HAMBRICK, MASON, 1984; LEWIN, STEPHENS, 1994) e a tomada de decisão em nível estratégico (RAJAGOPALAN; RASHEED; DATTA, 1993; PAPADAKIS; BARWISE, 2002). Mesmo de forma mais abrangente como no caso da intenção estratégica, estes executivos em casos de sucesso promovem direção, motivação $e$ desafio (HAMEL; PRAHALAD, 1994), influenciando todo o desdobramento de ações em relação aos recursos e processos.

De forma a contribuir para a melhor compreensão do conhecimento até então adquirido sobre a tomada de decisão estratégica do alto escalão, este trabalho tem como objetivo analisar a estrutura intelectual que conecta teorias e autores que pesquisam a tomada de decisão estratégica do alto escalão de forma a propor uma agenda para pesquisa futura em tomada de decisão estratégica.

Foram identificados três clusters teóricos: as características do Top Management Team (TMT) e como estas influenciam a tomada de decisão; fatores ambientais e sua influcência na tomada de decisão, juntamente com a exposição de modelos e processos de tomada de decisão; conflito e consenso na tomada de decisão. A discussão permitiu ilustrar o atual estado do tema e suas lacunas e apresentar sugestões para pesquisas futuras.

Este artigo está organizado em quatro partes. A seguir a esta introdução, na segunda parte, é apresentada a revisão de literatura sobre o tema tomada de decisão. A terceira parte expõe a metodologia utilizada no estudo, apresentando a natureza e a abordagem da pesquisa, os métodos de pesquisa, a seleção $e$ classificação dos dados e fontes de pesquisa, a coleta e tratamento dos dados, a análise dos dados, e as limitações do estudo. Dando seguimento são apresentados os resultados que serão discutidos aprofundadamente na quinta parte, juntamente com o modelo teórico resultante e a concepção de uma proposta de agenda de estudos futuros. Por fim, são apresentadas as considerações finais sobre a tomada de decisão estratégica pelo Alto escalão.

\section{Revisão de Literatura}

A tomada de decisão estratégica é contingencial e complexa, influenciada diretamente por fatores como variabilidade do mercado, comportamento oportunístico, pela pressão de tempo, dentre outros (EINSENHARDT; ZBARACKI, 1992). Está relacionada ao sucesso das organizações bem como o rumo estratégico tomado pelas mesmas (KUNC; MORECROFT, 2010) e ao seu declínio. (SERRA; ALMEIDA; FERREIRA, 2012) 
Qualquer que seja a abordagem para criação ou interpretação da estratégia, a mesma envolve uma escolha, uma tomada de decisão (PORTER, 1991). Diversos estudos abordam a tomada de decisão, dentre os quais se pode citar, estudos acerca da influência da tomada de decisão no desempenho (FREDERICKSON, 1984; HOUGH; WHITE, 2003; KUNC; MORECROFT, 2010), estudos sobre alto escalão na tomada de decisão (HAMBRICK, 1981; HAMBRICK; MASON, 1984; HAMBRICK; FILKENSTEIN, 1987), estudos sobre a influência cognitiva na tomada de decisão (SCHWENK, 1986; DAS; TENG, 1999; HASELTON; NETTLE; ANDREWS, 2005), estudos sobre a pressão ambiental e o tempo na tomada de decisão (KOCHER; SUTTER, 2006). O desempenho das organizações e o rumo seguido parecem, assim, inexoravelmente ligados à tomada de decisão.

\subsection{Teoria do Alto escalão e a VBR}

Hoskisson et al. (1999) argumentaram que a perspectiva do alto escalão apresentada por Hambrick e Mason (1984) pode ser considerada uma teoria da administração estratégica. Hoskisson et al. afirmam que o resultado da organização é influenciado pelas características do principal executivo e de sua equipe próxima, estando estas características ligadas com as ações e direcionamentos estratégicos, que resultam em desempenho empresarial (MEDEIROS JR.; SERRA; FERREIRA, 2010). Assim, de acordo com Hoskisson et al. (1999), que revisam a evolução do estudo da administração estratégica, argumentam que tanto os trabalhos de liderança estratégica como os de decisão estratégica são um sub-tema da VBR, representada principalmente pela teoria do alto escalão proposta por Hambrick e Mason (1984).

Hoskisson et al. (1999, p. 440) vão ainda mais longe ao mencionar que os líderes estratégicos nas organizações podem ser considerados como "[...] potencialmente o único recurso da empresa". Desde a publicação do artigo de Hambrick e Mason (1984) acerca da perspectiva do alto escalão, que aborda que o desempenho e o rumo estratégico empresarial são em parte reflexos das características e escolha dos gestores de topo, outros autores têm adotado a perspectiva que a equipe executiva é um recurso estratégico (MILLER; TOULOUSE, 1986; FINKELSTEIN; HAMBRICK, 1988;
SMITH et al., 1994). Em especial, essas pesquisas têm focado a influência do alto escalão sobre o desdobramento e desenvolvimento dos recursos (BARNEY, 1991; PETERAF, 1993; AMIT; SCHOEMAKER, 1993). Outros autores observam como as decisões estratégicas da empresa são influenciadas pelo talento gerencial (SELZNICK, 1957; HAMBRICK; FINKELSTEIN, 1987), base, conhecimento, habilidades e estilos cognitivos do alto escalão (HAMBRICK; MASON, 1984). Um argumento que reforça esta afirmação foi exposto por Rumelt (1984), notando que os casos de empresas de sucesso estão ligados a relações especificas e únicas de recursos específicos, influenciadas pelo próprio alto escalão e sua ação de tomada de decisão gerencial.

Levando em consideração o conceito VRIN e a argumentação de Barney (1991), o alto escalão, ao criar e executar estratégias que possibilitam a exploração das oportunidades e/ou neutralização de ameaças, pode ser considerado um recurso valioso. De forma similar, embora os líderes possam ser formados, são raros (COLLINS, 2001). Os membros do alto escalão das empresas possuem capacidades sócio-cognitivas diferentes entre si, de fundo emocional e racional adquiridos durante a vida ou geneticamente, sendo dificilmente replicáveis, podendo ser consideradas como recursos que induzem heterogeneidade nos setores competitivos. (PRAHALAD; BETTY, 1986; GRANT, 1988)

$\mathrm{O}$ alto escalão toma decisões específicas que influenciam o desdobramento e desenvolvimento de recursos com impacto no atingir de vantagens sustentáveis (AMIT; SCHOEMAKER, 1993). A tomada de decisão é dependente do comportamento e assim como a implementação das ações estratégicas na organização também serão distintas, relacionando diretamente com a inimitabilidade (AMIT; SCHOEMAKER, 1993). Pode-se argumentar, na perspectiva de Rumelt (1984), que o alto escalão é capaz de isolar suas empresas da imitação por sua atuação de manutenção e acesso a clientes, ao direcionamento de inovações e à descoberta de oportunidades. A importância do executivo principal é abordada nos trabalhos de empreendedorismo de Gartner (1988), sustentando o argumento que tanto a empresa como o empreendedor são historicamente dependentes. Pode-se também estender para aspectos de complexidade social e ambiguidade causal, visto que não é fácil compreender a forma pela qual as 
decisões sobre recursos acontecem, ou como são os relacionamentos no alto escalão, e entre este e outros níveis hierárquicos. Esta é uma das possibilidades de pesquisa levantadas na nova abordagem de estratégica como prática, cujo foco está nas práticas sociais da criação estratégica. (VAARA; WHITTINGHTON, 2012)

Pelo que foi exposto fica clara a influência do alto escalão nas decisões e a importância da tomada de decisão estratégica para o desempenho das organizações. Avaliando mais de perto os trabalhos de Hambrick a partir da perspectiva do alto escalão, também fica claro o impacto do executivo principal e sua equipe no desempenho empresarial, e nas decisões estratégicas e rumo estratégico.

\subsection{Tomada de Decisão Estratégica no Alto Escalão}

A tomada de decisão está presente nos diversos níveis organizacionais e, possivelmente com maior saliência pelo impacto na organização, no nível estratégico (ELBANNA, 2006). Einsenhardt e Zbaracki (1992) definem as decisões estratégicas como as decisões importantes em termos das ações que determinam a alocação de recursos que comprometem e auxiliam os ajustes estratégicos. A tomada de decisão estratégica é contingencial e complexa, influenciada diretamente por fatores como variabilidade do mercado, comportamento oportunístico, pressão de tempo, dentre outros. (EINSENHARDT; ZBARACKI, 1992)

Elbanna (2006) realizou uma revisão sobre tomada de decisão estratégica. Argumenta que as decisões estratégicas têm caracter formal ou informal, que são da responsabilidade do alto escalão e que refletem a interação entre a organização e seu ambiente. Também, que as decisões estratégicas tratam de interesses que são essenciais à sobrevivência da organização, envolvendo uma grande diversidade de recursos organizacionais. Por fim, ele concluiu que as decisões estratégicas são iminentemente políticas, empregnadas de elevados níveis de incerteza, nem sempre refletem a melhor solução, cujo que resultado no desempenho é difícil de avaliar, e que pela sua natureza depois de tomadas são difíceis de reverter.

O processo de tomada de decisão é complexo, na medida em que envolve características organizacionais e questões acerca dos tomadores de decisão
(KEENEY, 2004). O estudo de revisão de literatura de Elbanna (2006) classificou as pesquisas sobre decisão estratégica em dois grupos: as que tratam conteúdo e as que se debruçam sobre o processo. As que tratam conteúdo focam temas como portfólio, diversificação, gestão e alinhamento estratégico. As que se debruçam sobre o processo analisam os fatores envolvidos no próprio processo.

O artigo de Hambrick e Mason (1984) foi um dos trabalhos iniciais relacionando as características dos tomadores de decisão, no caso o alto escalão, ao desempenho organizacional. Depois deste, uma série de trabalhos procuraram relacionar características do alto escalão com os resultados da organização. Destacam-se Hambrick, Cho e Chen (1996) e Friedrickson, Hambrick e Baumrin (1998) que avaliaram a relação entre o principal executivo e o conselho de administração. Filkelstein e Hambrick (1988), Filkelstein e Hambrick (1989) e Sanders e Hambrick (2007) no estudo da influência da remuneração dos executivos principais no desempenho das organizações. Hambrick e Fukutomi (1991) e Henderson, Miller e Hambrick (2006) sobre a evolução do comportamento do CEO durante seu mandato.

Hambrick e D'Aveni (1992) e Hambrick (1995) mencionam acerca do impacto da fragmentação do alto escalão nas estratégias e nos resultados das organizações. E, Hayward e Hambrick (1997) e Hiller e Hambrick (2005) estudaram como a personalidade do CEO define a escolha estratégica.

Uma parte substancial da literatura sobre a tomada de decisão está vinculada a cognição dos indivíduos decisores, e seus vieses cognitivos. Eisenhardt e Zbaracki (1992) defendem a consideração da cognição, da intuição e dos insights dos decisores no processo decisório, argumentando que estes complementam a racionalidade humana. Os vieses cognitivos são efeitos resultantes da cognição, de observação pessoal (por exemplo, falhas de cálculo, de atribuição social e de memória) comuns em todos os seres humanos (HASELTON; NEETLE; ANDREWS, 2005). Diversos autores analisaram os efeitos adversos dos vieses cognitivos nos processos de tomada de decisão. (SCHWENK, 1986; DAS; TENG, 1999)

Dentre os fatores que afetam a cognição dos decisores, é possível citar a complexidade da tomada de decisão. Simon (1957) explorou os limites da to- 
mada de decisão humana em ambientes dinâmicos e complexos. Devido à complexidade e ao dinamismo ambiental em torno da tomada de decisão, sugeriu que os seres humanos não são capazes de agir de forma totalmente racional, e que essa incapacidade resulta em um estado geral de satisficing, na qual soluções não ideais são escolhidas ao atenderem requisitos mínimos da tomada de decisão.

Isso ocorre devido à racionalidade limitada dos seres humanos, que não são, muitas vezes, mentalmente capazes de avaliar todas as consequências potenciais das decisões tomadas. Assim, quando há muitas questões com resultados incertos e instáveis, a análise das possíveis decisões e seus resultados renderia conclusões muito complexas e incertas. (SIGGELKO; RIVKIN, 2005)

A tomada de decisão seria o equivalente a escolher um resultado potencial de dentro uma lata de lixo (ver o conceito de garbage can de Cohen et al. (1972)). A complexidade refere-se ao número e interdependências dos componentes que existem em qualquer processo de tomada de decisão. Alguns autores argumentam que quanto maior as interdependências entre as variáveis, maior será o nível de complexidade envolvido na tomada de decisão. (MISCHEN; JACKSON, 2008)

Outro fator preponderante influenciador na tomada de decisão é o tempo. Diante da pressão de tempo, o tomador de decisão está propenso a usar excessivamente analogias simplificadas para conceitualizar uma situação complexa (RAHMAN; DE FEIS, 2009). Muitas variáveis, tempo limitado, e os concorrentes fazem com que a tomada de decisão seja complexa, instável e arriscada (SIMON, 1979). Além disso, em ambientes em rápida mudança, a pressão do tempo é uma força proeminente (SIMON, 1979; KOCHER; SUTTER, 2006). Em um mercado competitivo, tomar a decisão certa, por si só não dá a uma empresa vantagem competitiva, mas tomar uma decisão certa e rápida pode levar a vantagem competitiva temporária. (PORTER; MILLAR, 1985)

O ambiente empresarial em que a organização está inserida é passível de afetar e/ou restringir a tomada de decisão (RAHMAN; DE FEIS, 2009). Diversos estudos investigam os potenciais efeitos de moderação dos fatores ambientais sobre a relação entre as dimen- sões do processo de decisão e resultados econômicos (FREDERICKSON, 1984; HOUGH; WHITE, 2003) e como o comportamento político afeta os processos de decisão estratégica dos CEOs em ambientes dinâmicos (EINSENHARDT; BOURGEOIS, 1988). Por exemplo, Bougeois e Eisenhardt (1988) concluíram que em ambientes de negócios dinâmicos, é imperativo que a tomada de decisão seja cuidadosa, porém rápida, e que esses ambientes demandam um TMT (top management team - time de alto escalão) poderoso e decisivo, que persiga o risco e a inovação, porém com execução segura e incremental. Em outro artigo, Einsenhardt (1989) aborda que em ambientes com alta dinâmica, os tomadores de decisão mais rápidos usam mais e não menos informações que os tomadores de decisões mais lentos, assim como também usam mais e não menos alternativas.

\section{Metodologia}

Nesta seção são apresentados os procedimentos metodologicos, no caso, o levantamento bibliométrico, a escolha das bases de dados e o tratamento dos dados encontrados na pesquisa.

\subsection{Método de Pesquisa}

Foi realizado um levantamento bibliométrico, a fim de fundamentar a revisão e o entendimento da literatura. Os estudos bibliométricos têm sido amplamente utilizados para compreender a literatura existente e, nomeadamente, identificar a estrutura intelectual de uma área de estudo. Visam entender a influência do trabalho de um autor (FERREIRA, 2012; FERREIRA; SERRA; ALMEIDA, 2012) e ou uma disciplina (RAMOS-RODRIGUEZ; RUIZ-NAVARRO, 2004). Por exemplo, Moran et al. (2010) fizeram um estudo bibliométrico sobre alianças estratégicas, Guillera et al (2013) verificaram a evolução da pesquisa de psicologia ao redor do mundo. Ferreira, Santos, Almeida e Serra (2012) estudaram a evolução da investigação em fusões e aquisições por meio de uma pesquisa bibliométrica, que englobou um período de dez anos nas seis revistas de maior reputação no campo da estratégia. 


\subsection{Seleção e Classificação dos Dados e Bases de Dados}

A base de dados escolhida para a pesquisa de periódicos internacionais foi a ISI Web of Science, pertencente à plataforma da Thomson Reuters Web of Knowledge e considerada uma das bases mais completas. (QUALIS, 2010)

\subsection{Coleta e Tratamento dos Dados}

Para a coleta dos dados, foi necessário determinar o intervalo de tempo a ser pesquisado na base, as palavras-chave a utilizar, os índices da base, os tipos de documentos pesquisados, $e$ finalmente as áreas pesquisadas. Quanto ao intervalo de tempo a ser pesquisado, escolheu-se pesquisar em todos os anos disponíveis na base, com o intuito de compor uma amostra relevante que incluisse o maior número possível de artigos que possibilitaria uma análise longitudinal da evolução temporal dos trabalhos desenvolvidos sobre o tema de tomada de decisão.

A pesquisa foi conduzida com a palavra-chave "decision making" seguida de uma palavra-chave referente ao tomador de decisão. O asterisco "*" ao final da palavra-chave refere-se à pesquisa de quaisquer variações possíveis da palavra em relação à última letra, incluindo plurais, singulares e variações, como por exemplo, "board" e "boards". Foram as seguintes as palavras que foram combinadas: tmt, top management, $\mathrm{CEO}$, board, uper management, higher management, dean, sênior, diretor.

Na escolha dos índices da base Web Of Science, foram selecionados todos os quatro índices disponíveis, assegurando o achado da maior quantidade possível de trabalhos, e a possível escolha das áreas a ser filtrar os artigos posteriormente. Escolheu-se também pesquisar somente artigos, excluindo notas, revisões de livro, $e$ trabalhos de congresso. Por fim, foi aplicado um filtro de modo a incluir apenas periódicos de Management e Business. Estes procedimentos resultaram na identificação de 961 artigos.

Para a obtenção da amostra final, procedeu-se à leitura dos títulos, resumos e palavras-chave dos 961 artigos. Esse procedimento permitiu eliminar os artigos que não refletissem o tema pesquisado. Assim, obteve- -se um total final de 277 artigos que é a amostra final para ao estudo.

Vale ressaltar que, apesar de a pesquisa contemplar toda a base, os periódicos dos artigos publicados possuem, em geral, fator de impacto relevante. Foram 78 periódicos com artigos selecionados. Todos tinham fator de impacto acima de 0,2 (Qualis A2) e, do total $68(87 \%)$ com fator de impacto acima de 1 (Qualis A1). (CAPES, 2010)

\subsection{Análise dos Dados}

Utilizando o software Bibexcel - esse software é de livre acesso em www.umu.se/inforsk/Bibexcel - foi possível gerar a um conjunto de elementos para análise: a tabela de frequência das referências contidas dos artigos e gerar a matriz de cocitação necessária para a criação dos mapas, ou redes, de cocitação. A partir das matrizes de cocitação, foram gerados as redes de cocitação, utilizando-se o software SPSS versão 18.

Com as tabelas de frequência de citações contidas nos artigos e com os mapas de cocitação, foi possível analisar como estão fundamentados os artigos da amostra. Mais especificamente, foi possível analisar, pela frequência de citação das referencias, quais os trabalhos mais utilizados na fundamentação conceitual em tomada de decisão. A visualização das redes de cocitação permitiu identificar como os trabalhos mais citados estão relacionados na fundamentação do tema, ou seja, a estrutura intelectual, e inferir como estão estruturados os artigos de tomada de decisão.

\section{Resultados}

\subsection{Resultados de Citação e Cocitação}

A apresentação dos resultados mostra os resultados da análise bibliométrica e de cocitação. No caso, as referências mais influentes, os trabalhos que sustentam e suportam os estudos e os mapas de co-citação, com os quais se pode visualizar os clusters do tema.

\subsubsection{Referências mais Citadas}

Com base nos 277 artigos da amostra, selecionados na base ISI Web Of Science, elaborou-se a Tabela 2, 
com os 26 trabalhos mais citados. Como critério de corte foram escolhidos trabalhos que tivessem 25 ou mais citações. Pode-se observar na leitura da Tabela 2 que o trabalho de Hambrick e Mason (1984) é o mais citado, com 83 citações, o que significa que foi citado em $30 \%$ da amostra. O segundo artigo mais citado é o artigo de Amason (1996), com 66 citações (ou 24\% dos 277 artigos), e o terceiro é um artigo de Eisenhardt (1989), com 53 citações (19\%). Os trabalhos da Tabela 1 foram utilizados nos mapas de cocitação com 26 referências (Figura 1).

Tabela 1: Os 26 trabalhos mais citados nas referências dos 277 trabalhos

\begin{tabular}{|c|c|c|c|}
\hline$\#$ & \# DE CITAÇÕES & REFERÊNCIAS & \% DE CITAÇÕES \\
\hline 1 & 83 & $\begin{array}{l}\text { Hambrick, D.\& Mason, P. (1984). Upper echelons: The organization as a reflection } \\
\text { of its top managers. Academy of Management Review, v. 9, n. 2, p. 193-206. }\end{array}$ & $28,92 \%$ \\
\hline 2 & 66 & $\begin{array}{l}\text { Amason, A. (1996) Distinguishing the effects of funcional and dysfuncional } \\
\text { conflict on strategic decision making: Resolving a paradox for top management } \\
\text { teams. Academy of Management Journal, v. 39, p. } 123148 .\end{array}$ & $23,00 \%$ \\
\hline 3 & 53 & $\begin{array}{l}\text { Eisenhardt, K. (1989) Making fast strategic decisions in high-velocity } \\
\text { environments. Academy of Management Journal, v. 32, n. 3, p. 543-576 }\end{array}$ & $18,47 \%$ \\
\hline 4 & 48 & $\begin{array}{l}\text { Bantel, K. \& Jackson, S. (1989). Top management and innovations } \\
\text { in banking: Does the composition of the top team make a difference. } \\
\text { Strategic Management Journal, v. 10, p. 107-124. }\end{array}$ & $16,72 \%$ \\
\hline 5 & 41 & $\begin{array}{l}\text { Eisenhardt, K. \& Bourgeois, L. (1988) Politics of strategic decision } \\
\text { making in high-velocity environments: Toward a midrange theory. } \\
\text { Academy of Management Journal, v. 31, n. 4, p. 737-770. }\end{array}$ & $14,29 \%$ \\
\hline 6 & 40 & $\begin{array}{l}\text { Jehn, K. (1995) A multimethod examination of the benefits and detriments of } \\
\text { intragroup conflict. Administrative Science Quarterly, v. 40, n. 2, p. 256-282. }\end{array}$ & $13,94 \%$ \\
\hline 7 & 39 & $\begin{array}{l}\text { Wiersema, M. \& Bantel, K. (1992) Top management team demography and corporate } \\
\text { strategic change. Academy of Management Journal, v. 35, n. 1, p.91-121. }\end{array}$ & $13,59 \%$ \\
\hline 8 & 38 & $\begin{array}{l}\text { Finkelstein, S. \& Hambrick, D. (1996) Strategic leadership: Top executives } \\
\text { andtTheir effects on organizations. New York: West Publishing Company. }\end{array}$ & $13,24 \%$ \\
\hline 9 & 37 & $\begin{array}{l}\text { Mintzberg, H.; Raisinghani, D \& Theorêt, A. (1976) The structure of "unstructure" } \\
\text { decision processes. Administrative Science Quartely, v. 21, n. 2, p. 246-275. }\end{array}$ & $12,89 \%$ \\
\hline 10 & 34 & $\begin{array}{l}\text { Pelled, L; Eisenhardt, K. \& Xin, K. (1999) Exploring the black box: an analysis of work group } \\
\text { diversity, conflict, and performance. Administrative Science Quarterly, v. 44, n. 1, p. 1-28. }\end{array}$ & $11,85 \%$ \\
\hline 11 & 34 & $\begin{array}{l}\text { Eisenhardt, K. \& Zbaracki, M. (1992) Strategic decision making. } \\
\text { Strategic Management Journal, v. 13, p. 17-37. }\end{array}$ & $11,85 \%$ \\
\hline 12 & 32 & $\begin{array}{l}\text { Simons, T.; Pelled, L. \& Smith, K. (1999). Making use of difference: } \\
\text { Diversity, debate and decision comprehensiveness in top management } \\
\text { teams. Academy Of Management Journal, v. 42, p. } 662673 .\end{array}$ & $11,15 \%$ \\
\hline 13 & 32 & $\begin{array}{l}\text { Smith, K. G.; Smith, K. A.; Olian, J. D.; Sims Jr, H. P.; O`Bannon, D. P. \& Scully, J. A. } \\
\text { (1994) Top management team demography and process: The role of social integration and } \\
\text { communication. Administrative Science Quarterly, New York, v. 39, n. 3, p. 412-438. }\end{array}$ & $11,15 \%$ \\
\hline 14 & 32 & $\begin{array}{l}\text { Cyert, R. \& March, J. (1963) A Behavioral theory of the } \\
\text { firm. Prentice Hall, Englewood Cliffs, NJ. }\end{array}$ & $11,15 \%$ \\
\hline 15 & 30 & $\begin{array}{l}\text { Finkelstein, S. \& Hambrick, D. (1990) Top management team tenure } \\
\text { and organizational outcomes: The Moderating Role Of Managerial } \\
\text { Discretion. Administrative Science Quarterly, v. 35, p. 484-503. }\end{array}$ & $10,45 \%$ \\
\hline 16 & 29 & $\begin{array}{l}\text { Fama, E.; Jensen, M. (1983) Separation of ownership and control. } \\
\text { Journal Of Law And Economics, v. 26, p. } 301 .\end{array}$ & $10,10 \%$ \\
\hline 17 & 28 & Porter, M. (1980) Competitive Strategy. Free Press: New York. & $9,76 \%$ \\
\hline
\end{tabular}




\begin{tabular}{|c|c|c|c|}
\hline \# & \# DE CITAÇÕES & REFERÊNCIAS & \% DE CITAÇÕES \\
\hline 18 & 27 & $\begin{array}{l}\text { Hambrick, d.; Cho, T. \& Chen, M. (1996) The influence of top management } \\
\text { team heterogeneity on firms' competitive moves. Administrative } \\
\text { Science Quarterly, New York, v. 41, n. 4, p. 659-684. }\end{array}$ & $9,41 \%$ \\
\hline 19 & 27 & March, J.; Guetzkow, H. \& Simon H. (1958) Organizations. New York: John Wiley \& Sons. & $9,41 \%$ \\
\hline 20 & 26 & $\begin{array}{l}\text { Michel, J. \& Hambrick, D. (1992) Diversification posture and the characteristics of the top } \\
\text { management team. Academy Of Management Journal, New York, v. 35, n. 1, p. 9-37. }\end{array}$ & $9,06 \%$ \\
\hline 21 & 26 & $\begin{array}{l}\text { Forbes, D \& Milliken, F. (1999) Cognition and corporate governance: } \\
\text { Understanding boards of directors as strategic decision-making groups. } \\
\text { Academy Of Management Review, v. } 24, \text { n. 3, p. } 489-505 .\end{array}$ & $9,06 \%$ \\
\hline 22 & 26 & $\begin{array}{l}\text { Hambrick, D. (1994) Top management groups: A conceptual integration and } \\
\text { reconsideration of the "team" label. Research In Organization Behaviour, v. 16, p.171-214. }\end{array}$ & $9,06 \%$ \\
\hline 23 & 26 & $\begin{array}{l}\text { Bourgeois, L. \& Eisenhardt, K. (1988) Strategic decision processes } \\
\text { in high velocity environments: Four cases in the microcomputer } \\
\text { industry. Management Science, v. 34, p. } 816-835\end{array}$ & $9,06 \%$ \\
\hline 24 & 25 & $\begin{array}{l}\text { Schweiger, D.; Sandberg, W. \& Ragan, J. (1989). Experiential effects of } \\
\text { dialectical inquiry, Devil's advocacy, and consensus approaches to strategic } \\
\text { decision-making. Academy Of Management Journal, v. 32, p. 745-772. }\end{array}$ & $8,71 \%$ \\
\hline 25 & 25 & $\begin{array}{l}\text { Murray, A. (1989) Top Management group heterogeneity and firm } \\
\text { performance. Strategic Management Journal, v. 10, p. 125-141. }\end{array}$ & $8,71 \%$ \\
\hline 26 & 25 & $\begin{array}{l}\text { Aiken, L. \& West, S. (1991) Multiple regression: Testing and } \\
\text { interpreting interactions. Newbury Park, CA: Sage. }\end{array}$ & $8,71 \%$ \\
\hline
\end{tabular}

Fonte: Elaborada pelos autores deste artigo

\subsubsection{Resultados de Cocitação}

O map de cocitação representados na Figura 1 mostra visualmente a proximidade intelectual entre os trabalhos. Ao ler a figura é importante prestar atenção que a maior proximidade entre os trabalhos significa que os trabalhos foram mais frequentemente citados juntamente em outros trabalhos. O diâmetro do círculo é proporcional à frequência de citação. Essa forma de representação visual facilita a compreensão das relações entre os trabalhos representados nos mapas, evidenciando a estrutura das ligações intelectuais entre os trabalhos no contexto específico da tomada de decisão estratégica.

Para identificação da existência de clusters, todos os artigos que compõem o mapa foram lidos e analisados. Da análise resultam três clusters. Um Cluster
\#1, denominado Características do TMT ("top management team"), que está composto de trabalhos que abordam as características e composição dos gestores de topo em relação à qualidade ou desempenho de sua tomada de decisão; um Cluster \#2, denominado Ambiente, Modelos e Processos de Tomada de Decisão, que apresenta, como explicitado pelo título, os modelos e os processos para tomada de decisão, juntamente com características e fatores ambientais que afetam a tomada de decisão; e, por fim, o Cluster \#3, denominado Conflito e Consenso, que aborda especificamente os aspectos relacionados aos conflitos e o consenso na tomada de decisão. Os clusters serão detalhados a seguir, a partir da descrição dos trabalhos que os compõem em ordem cronológica, de forma a possibilitar uma análise posterior com os demais resultados gerados neste estudo. 


\section{Object Points}

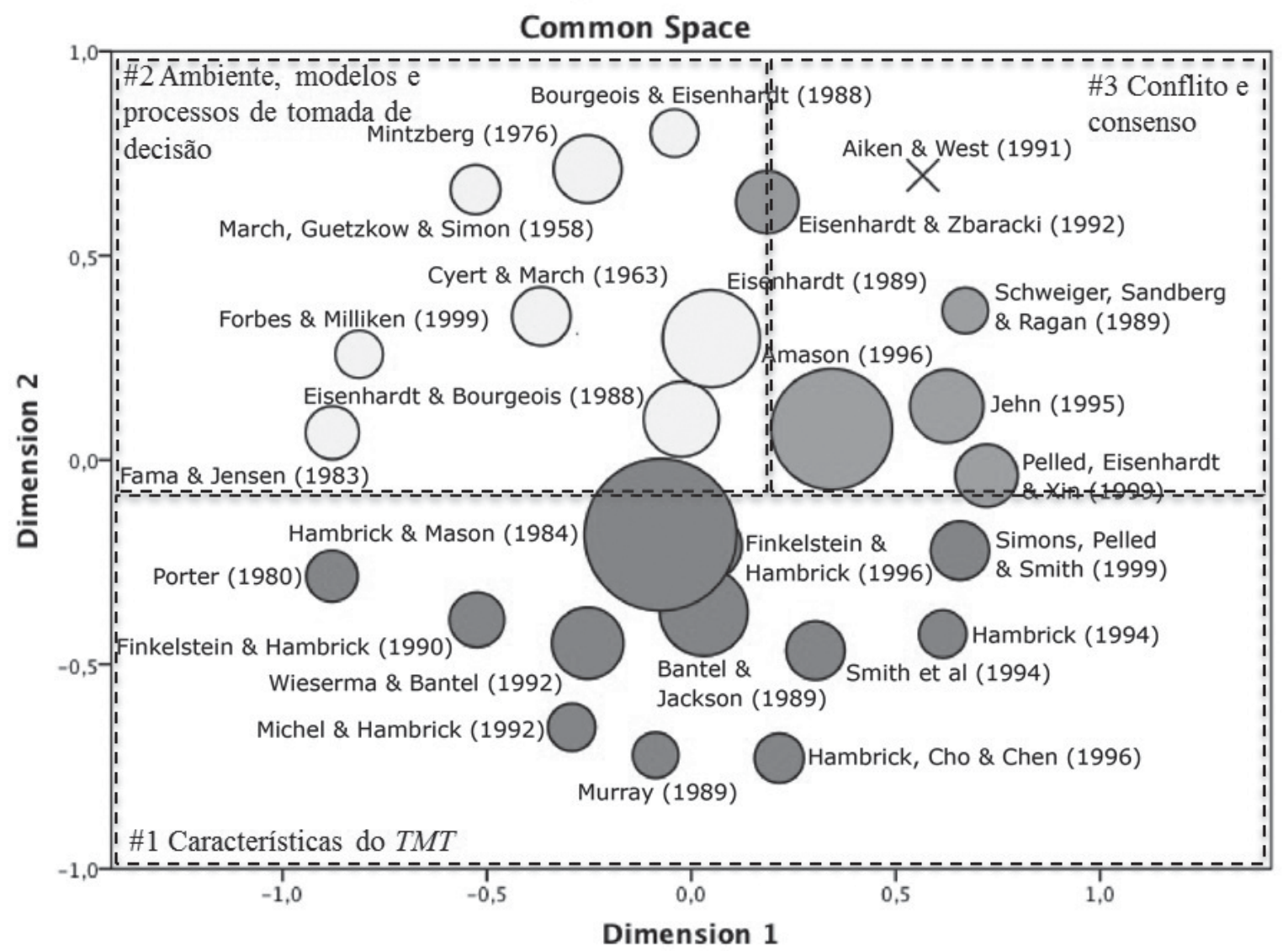

Figura 1: Mapa de cocitação das 26 referências

Fonte: Elaborado pelos autores deste artigo

\subsubsection{Apresentação do Cluster \#1}

O cluster \# 1 trabalha as características do TMT e tem como referencia fundamental o trabalho de Hambrick e Mason (1984). Este trabalho, com o título Upper echelons: The organization as a reflection of its top managers é considerado o trabalho seminal da Teoria do Alto Escalão (HOSKISSON et al., 1999; PAPADAKIS; BARWISE, 2002). Neste artigo, os autores apresentam a perspectiva do Alto Escalão, argumentando que são as escolhas estratégicas, oriundas das decisões do TMT, e influenciadas pelas características deste grupo que impactam os resultados das organizações. As características do TMT são classificadas por Hambrick e Mason (1984) em perceptíveis e psicológicas. As características perceptíveis, segundo os autores auxiliam na formação das características psicológicas e incluem aspectos fáceis de identificar e mensuráveis (por exemplo, idade, formação, trilha funcional, heterogeneidade, dentre outras). As características psicológicas, além da base cognitiva, podem ser também ligadas aos valores pessoais, sendo assim mais difíceis de medir.

Bantel e Jackson (1989) investigaram a relação entre a composição dão alto escalão e a capacidade de inovar. Bantel e Jackson testaram o nível de recursos cognitivos e características demográficas do $\mathrm{CEO}$ e da TMT, mostrando que os recursos cognitivos no processo de tomada de decisão e a diversidade de visões são importantes para a inovação. Murray (1989) estudou o impacto da heterogeneidade da composição da TMT no desempenho em relação às condições ambientais. Mostrou como a heterogeneidade da equipe afeta o desempenho de duas maneiras: pelo baixo processo de socialização pode implicar na maior exploração de alternativas estratégicas e, por uma maior amplitude de backgrounds, pode auxiliar na identificação de novas estratégias. 
Em Finkenstein e Hambrick (1990), os autores estudaram a relação entre o tempo de mandato (tenure) da TMT em relação ao desempenho. Os resultados da pesquisa mostram que o tempo de mandato da TMT tem uma influência grande sobre aspectos como persistência estratégica, conformidade estratégica e de resultado. Outro resultado é a necessidade de considerar o papel moderador da discricionariedade (managerial discretion) em trabalhos futuros.

Em seu artigo de 1994, Hambrick tenta integrar a pesquisa do TMT procurando dar coerência e síntese, dez anos depois de seu artigo seminal (HAMBRICK; MASON, 1984). Sobretudo, neste artigo, ele apresenta do conceito de integração comportamental para os grupos de TMT (HAMBRICK, 1994). O autor descreve as várias "forças centrífugas" que tendem a diminuir a integração comportamental do grupo e discute as implicações desta integração para a adaptação organizacional e efetividade do grupo. Essas "forças centrífugas" são: o tamanho da organização, que em grandes organizações reduz a integração; a diversificação, que tende a uma menor integração do TMT; a estratégia do negócio que considerando a tipologia de Miles e Snow (1978), em organizações que prospectam, com maior alteração de produtos em relação às oportunidades, a integração é maior; organizational slack (CYERT; MARCH, 1963), a diferença entre os recursos da organização e a combinação de demanda sobre eles (COHEN; MARCH; OLSEN, 1972, p. 12), no qual o slack moderado ou o baixo proporcionam maior integração; e dinamismo ambiental, quanto mais dinâmico o ambiente, maior a integração do TMT.

Smith et al. (1994) testaram três modelos alternativos para avaliar os efeitos da demografia e os processos de relacionamento do TMT no desempenho organizacional; num modelo somente a demografia influencia; o segundo sofre influência principalmente do processo; no terceiro modelo, interveniente, são as características demográficas influenciando o processo. Os resultados mostraram que o processo tem efeito direto sobre o desempenho, embora a demografia possa ter alguns impactos diretos e indiretos. Wiersema e Bantel (1992) estudaram aspectos demográficos da TMT focando o relacionamento entre a demografia da TMT e a mudança estratégica corporativa, medida pela diversificação das 500 maiores da revista Fortune. Em seu estudo concluíram que as TMTs com maior pro- pensão à mudança, caraterizam-se por menor média de idade, menor tempo de mandato organizacional, maior nível de educação, maior heterogeneidade de especialização educacional e maior treinamento acadêmico em ciências.

Michel e Hambrick (1992) estudaram a relação entre a postura de diversificação das empresas e a integração entre as unidades de negócio a partir do TMT. Os autores concluíram que quanto maior a necessidade de interdependência imposta pela estratégia de diversificação da empresa, maior a necessidade do TMT operar com uma base ampla.

Hambrick, Cho e Chen (1996) estudaram a influência da heterogeneidade da TMT em relação aos movimentos competitivos da empresa. Suas conclusões apontam que a heterogeneidade da TMT está associada positivamente com a propensão e importância da empresa em agir, com a importância do escopo de resposta e resultado em ambientes turbulentos. Desse modo, estaria associada negativamente com a velocidade de execução da ação e de geração de resposta.

No livro Strategic Leadership, Finkelstein e Hambrick (1996) apresentam uma revisão de estudos sobre o tema do alto escaláo. No conjunto de artigos que compõem o livro, os autores justificam o estudo do alto escalão pelo fato do tomador de decisão ser importante.

Simons, Pelled e Smith (1999) estudaram como as variedades da diversidade e do debate de TMT podem influenciar o desempenho financeiro de uma empresa. Ao contrário de outros estudos, os autores revelaram que o debate aumenta a tendência para a diversidade e para o desempenho da TMT. O processo de interação da TMT age como moderador da diversidade.

Embora o trabalho de Porter (1980) apareça no cluster, ele não é considerado como uma contribuição direta para a teoria do alto escalão.

\subsubsection{Apresentação do Cluster \#2}

O trabalho de Eisenhardt e Zbaraki (1992) influencia tanto o cluster \#2 como o cluster \#3, pois se trata de uma revisão de literatura sobre a tomada de decisão estratégica. A revisão se baseou nos paradigmas teóricos dominantes que seriam racionalidade limitada, a política e o poder e "lata de lixo" (garbage 
can). Na revisão, os autores identificaram os debates emergentes na época. Os autores concluíram que os tomadores de decisão são parcialmente racionais, que o poder tem preponderância sobre a escolha e que a sorte é importante.

O livro Organizations, de March, Guetzkow e Simon (1958), mais referenciado como March e Simon (1958), é considerado a base para os estudos relacionados à tomada de decisão. Em seu livro, os autores assumem o ponto de vista do tomador de decisões como um processador de informação, sendo este trabalho influente na obra posterior de Cyert $e$ March (1963).

A obra de Cyert e March (1963) é considerada como um dos trabalhos seminais da abordagem comportamental da empresa. O trabalho procura explicar como a decisão acontece no ambiente da empresa $e$ fundamenta-se no conceito de racionalidade limitada. Cyert e March (1963) argumentaram que as prioridades e informações podem ser inconsistentes ao longo do tempo, através das pessoas na organização, gerando conflitos potenciais. Os autores propuseram que as empresas satisfazem-se com seus resultados (satisfacing) no lugar de otimizá-los. Também apresentam o processo de tomada de decisão a partir dos objetivos da TMT, que acontecem tanto no nível da TMT como nos níveis gerenciais inferiores.

Mintzberg, Raisinghani e Théorêt (1976) estudaram 25 processos de tomada de decisão estratégica propuseram um método para tomada de decisões não programadas. O método envolve 12 elementos, três fases centrais, três conjuntos de rotinas de suporte $e$ três conjuntos de fatores dinâmicos propostos pelos autores.

Fama e Jensen (1983) argumentam que o mecanismo de controle ligado à tomada de decisões nas organizações é o TMT que lida com o problema da agência causado pela separação entre a propriedade e o controle.

Einsenhardt e Bourgeois (1988) abordam como o comportamento político pode afetar os processos de decisão estratégica de CEOs em um mercado dinâmico. Apresentam uma proposta teórica ligando poder, comportamento político e desempenho. Concluem que os comportamentos políticos vêm da centralização de poder e que CEOs autocráticos se engajam em política e geram o mesmo comportamento nos seus subor- dinados. Concluem também que o comportamento político é organizado em coalizões estáveis com base nas características demográficas e que esse comportamento está usualmente associado a desempenho ruim.

Bougeois e Eisenhardt (1988), na mesma base de empresas do artigo anterior, concluíram que, em ambientes de negócios dinâmicos, é imperativo que a tomada de decisão seja mais cuidadosa, porém rápida. Argumentam também que esses ambientes demandam um TMT poderoso e decisivo, que persiga o risco e a inovação, porém com execução segura e incremental. Continuando o trabalho Eisenhardt (1989) aborda que em ambientes dinâmicos, tomadores de decisão rápidos usam mais e não menos informações que os tomadores de decisões lentos, assim como também usam mais e não menos alternativas. A resolução de conflitos e integração entre as decisões estratégicas e os planos táticos são críticos.

Forbes e Milliken (1999) propõem um modelo de processos de tomada de decisão do TMT, que integra a literatura de dinâmica de grupos e da efetividade de trabalho grupal.

\subsubsection{Apresentação do Cluster \#3}

Como no cluster anterior, o artigo de Eisenhardt e Zbaraki (1992) também tem influência no cluster \#3. Schweiger, Sandberg e Ragan (1989) compararam em estudo de laboratório a efetividade de três abordagens para tomada de decisão estratégica em grupo: inquérito dialético, advocacia do diabo e consenso. $\mathrm{O}$ uso de inquérito dialético e advocacia do diabo geraram decisões de maior qualidade, porém com menor aceitação das decisões do grupo em relação ao consenso.

Jehn (1995) investigou situações em que o conflito poderia ser benéfico e concluiu que isso depende do tipo de conflito, da estrutura do grupo em relação ao tipo de tarefa, da interdependência da tarefa e das normas do grupo. A autora mostrou que conflitos de relacionamento e de tarefa são, em geral, negativamente associados com a satisfação dos indivíduos, com a afeição por outros membros do grupo e com a pretensão em continuar a permanecer no grupo. Entretanto, em grupos envolvidos em tarefas não rotineiras, o estudo reporta que o conflito de tarefa pode não ter um efeito ruim, podendo ser até mesmo positivo. 
Amason (1996), o artigo mais referenciado deste grupo, estudou o conflito em TMT. Seus resultados apontam que o conflito cognitivo deve ser encorajado enquanto o conflito afetivo deve ser evitado. Se o conflito acontecer desta forma, a tomada de decisão terá maior qualidade e será mais efetiva, pois existirão maiores níveis de consenso e de aceitação afetiva.

Pelled, Eisenhardt e Xin (1999) propuseram um modelo integrado dos relacionamentos entre diversidade, conflito e desempenho. Os resultados mostraram que a diversidade molda o conflito $e$ o conflito molda o desempenho. A diversidade funcional aumenta o conflito de tarefa e a existência de muitos tipos de diversidade acarretam em conflito emocional. As diversidades de raça e de tempo na função foram positivamente associadas com o conflito emocional, enquanto a diversidade de idade foi negativamente associada com esse conflito. A execução de tarefas rotineiras e a longevidade do grupo moderaram estas relações. Os resultados também mostraram que o conflito da tarefa tem efeitos mais favoráveis no desempenho cognitivo de tarefa que o conflito emocional.

Embora apareça na área do terceiro cluster, o livro de Aiken e West (1991) foi descartado por se tratar de um livro de estatística, indicando a presença de muitos artigos empíricos quantitativos.

\section{Discussão}

Foram identificados de três clusters que emergem da análise dos mapas de cocitação: cluster \#1 - Características do TMT, cluster \#2 - Ambiente, modelos e processos de tomada de decisão e cluster \#3 - Conflito e consenso. A análise dos clusters encontrados nos mapas de cocitação proporcionou a criação de um quadro conceitual sobre tomada de decisão, apresentado na Figura 2.

O quadro conceitual está dividido em três clusters teóricos, seguindo a divisão apresentada nos mapas de cocitação. O cluster \#1, denominado Características do TMT, aborda trabalhos sobre características do TMT e a relação delas com o desempenho. Esse cluster foi divido em dois subclusters, características perceptíveis e características psicológicas. O autor mais referênciado é Donald Hambrick, refenciado no cluster com uma estrela.
O cluster \#2 apresenta trabalhos sobre o ambiente em que são tomadas as decisões, juntamente com modelos e processos sobre tomada de decisão. Sua designação é Ambiente, modelos e processos de tomada de decisão. Esse cluster apresenta dois subclusters, um com os trabalhos sobre o ambiente, o outro sobre modelos e processos decisórios. Kathleen Eisenhardt é autora ou coautora de todos os trabalhos apresentados no subcluster Ambiente,

No terceiro cluster, chamado de conflito e consenso, estão representados os trabalhos sobre como o conflito, grupal ou individual, afetam o processo de tomada de decisão. É um cluster emergente, grande parte dos trabalhos referentes aos conflitos encontrados na amostra foi publicada depois do ano 2000. O autor mais representativo deste cluster é Allen Amason, seu artigo de 1996 é um dos primeiros a abordar de forma explícita como o conflito cognitivo e emocional afetam a tomada de decisão.

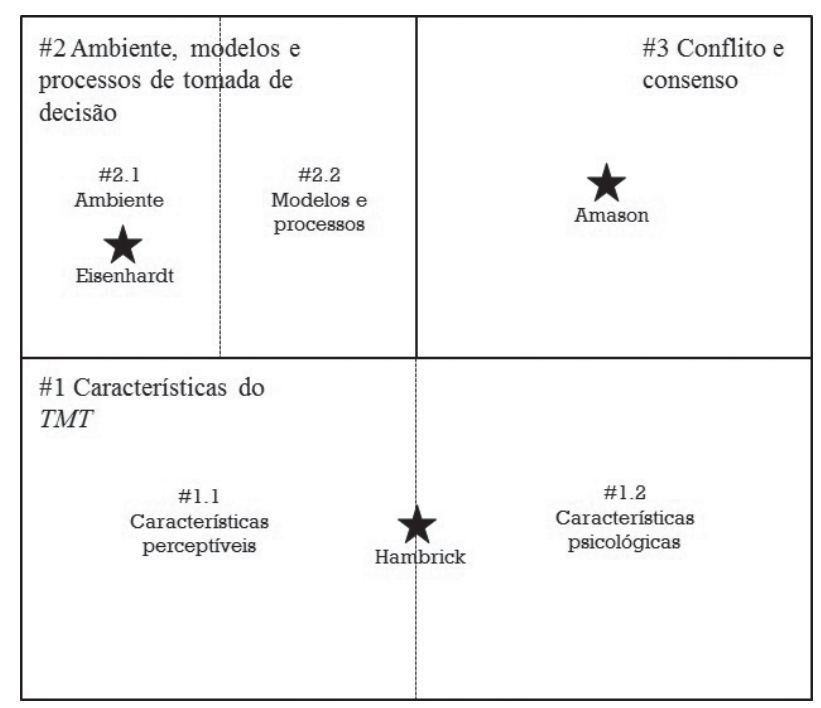

Figura 2: Quadro conceitual

Fonte: Elaborado pelos autores deste artigo

É importante ressaltar que a classificação proposta neste estudo é distinta da apresentada no trabalho de revisão de literatura de Eisenhardt e Zbaracki (1992). Eisenhardt e Zbaracki (1992) classificaram um conjunto de artigos de tomada de decisão de acordo com as abordagens teóricas de racionalidade limitada, política e poder e "lata de lixo". Por exemplo, alguns trabalhos que estão classificados como racionalidade limitada por Eisenhardt e Zbaraki (1992) neste estudo se encaixam no cluster \#2. O mesmo acontece em 
trabalhos incluidos no cluster \#3. Adicionalmente, nos clusters não foram encontrados trabalhos abordando o conceito de "lata de lixo", de forma que justificasse a análise por essa abordagem. Por fim, importa salientar que no trabalho de Eisenhardt e Zbaracki (1992) não existiam trabalhos referentes à teoria do alto escalão, a mais representativa neste estudo.

\subsection{Cluster \#1 - Características do TMT}

O cluster \#1 compreende artigos que avaliam as características do TMT e sua relação com o desempenho individual e organizacional. Estes artigos seguem principalmente os aspectos estabelecidos pela perspectiva do alto escalão proposta por Hambrick e Mason (1984) e as revisões e atualizações posteriores em Hambrick (1994) e Finkelstein e Hambrick (1996).

Os estudos sobre as características do TMT estão divididos em duas áreas: características perceptíveis e psicologicas. Essas áreas foram separadas em dois subclusters. A maioria dos trabalhos aborda características perceptíveis do TMT, como o trabalho de Murray (1989), Finkelstein e Hambrick (1990), Hambrick (1994), Smith et al. (1994), Mitchel e Hambrick (1992), Hambrick, Cho e Chen (1996) e Simons, Pelled e Smith (1999). Um fato que pode ajudar a explicar a predominância destes trabalhos no cluster \#1 é a possibilidade de medir as características perceptíveis utilizando dados secundários, por exemplo, dados da Fortune 500, relatórios anuais de empresas abertas e outras bases secundárias existentes.

Outro conjunto de trabalhos discorre sobre características psicológicas. É pequena a quantidade desses estudos no mapa de cocitação, possivelmente pelas dificuldades em medir características ligadas à cognição individual. Os recentes progressos tecnológicos e científicos aumentaram o acesso a equipamentos de medição e a diminuição do seus custos, proporcionando gradativamente um aumento na quantidade de estudos sobre cognição. Nesse grupo estão o trabalho de Bantel e Jackson (1989) e o livro de Finkeltein e Hambrick (1996). Hambrick e Mason (1994), ao propor estudos futuros sobre o alto escalão, citam a necessidade de medir a cognição dos indivíduos e cruzar tais estudos com caracteristicas perceptíveis. Eisenhardt e Zbaracki (1992) também argumentaram a importância dos estudos relacionados às características psicológicas ao propor racionalidade e racionalidade limitada como um tema de tomada de decisão.

\subsection{Cluster \#2 - Ambiente, Modelos e Processos de Tomada de Decisão}

O cluster \#2 engloba os trabalhos que se referem ao ambiente e como este afeta a tomada de decisão, juntamente com trabalhos sobre modelos e processos de tomada de decisão. É o segundo maior cluster na quantidade de trabalhos encontrados e é muito diverso quanto aos objetos de estudo. Este cluster foi dividido em dois subclusters. O primeiro, chamado de Ambiente, aborda o ambiente em que ocorre a tomada de decisão. São representantes deste sub cluster os trabalhos de Bourgeois e Eisenhardt (1988), Eisenhardt e Bourgeois (1988) e Eisenhardt (1989). Esses artigos abordam a tomada de decisão em ambientes dinâmicos e velozes.

Kathleen Eisenhardt é a autora com o maior número de trabalhos neste subcluster e no cluster \#2. Além disso, ela tem uma revisão de literatura sobre tomada de decisão que serve como arcabouço teórico para o cluster \#2 e \#3 e um trabalho que estuda conflito (PELLED; EISENHARDT; XIN; 1999), classificado no cluster \#3. A autora é prolífica no assunto tomada de decisão, podendo ser considera uma das mais importantes para o tema.

O segundo subcluster aborda trabalhos sobre modelos e processos de tomada de decisão. Nele estão contidos os trabahlos de March, Guetzkow e Simon (1958), Cyert e March (1963), Mintzberg, Raisinghani e Théorêt (1976), Fama e Jensen (1983) e Forbes e Miliken (1999). Há aqui três estudos que utilizam do conceito de racionalidade limitada e como ele afeta o processo de tomada de decisão. Os outros dois estudos, contidos no subcluster modelos e processos de tomada de decisão, não utilizam o conceito de racionalidade limitada, mas apresentam visões distintas sobre como funciona o processo de tomada de decisão.

\subsection{Cluster \#3 - Conflito e Consenso}

O cluster \#3 detém os artigos que abordam como o conflito afeta a tomada de decisão e o desempenho empresarial. A importância do tema foi apresentada por Eisenhardt (1992) em sua revisão de literatura 
sobre tomada de decisão, que apresentou em suas sugestões futuras a necessidade de se pesquisar como o conflito pode afetar o processo de tomada de decisão. Predominam neste cluster estudos qualitativos, que exigem maior rigor metodológico por parte dos pesquisadores e geram maior dificuldade em comprovar e generalizar a contribuição teórica. (SERRA; FIATES; FERREIRA, 2008)

Os trabalhos que compõem o cluster são Amason (1996), Schweiger, Sandberg e Ragan (1989), Jehn (1995) e Pelled, Eisenhardt e Xin (1999). Os estudos de Amason (1996), Jehn (1995) e Pelled, Eisenhardt e Xin (1999) apresentam uma tipologia similar de conflito. Separam os conflitos em: conflito de tarefa e conflito cognitivo, afetivo ou emocional. O conflito de tarefa ocorre quando existem divergências entre membros de um grupo sobre as tarefas que estão sendo realizadas. O conflito emocional ou afetivo lida com conflitos interpessoais de fundo afetivo, não resultantes da tarefa em que estão trabalhando. Um resultado comum aos três estudos citados acima é que o conflito emocional é prejudicial ao desempenho dos grupos, enquanto o conflito de tarefa é em geral benéfico ao desempenho, desde que não evolua para conflito emocional.

$\mathrm{O}$ autor com o trabalho mais referenciado foi Allen Amason, sendo o trabalho central sobre conflito no mapa de cocitação. É o trabalho mais utilizado como fundamentação para os estudos encontrados na amostra sobre conflito. Os outros três estudos do cluster foram referenciados em menor parte e de forma a complementar o estudo de Amason (1996).

\subsection{Agenda de Pesquisa Futura}

É necessário o aprofundamento do estudo sobre as características psicológicas dos invíduos tomadores de decisão. Autores como Hambrick e Mason (1994), ao propor estudos futuros sobre o alto escalão, citam a necessidade de medir a cognição dos indivíduos e cruzar tais estudos com caracteristicas perceptíveis. Ainda, Eisenhardt e Zbaracki (1992) também argumentaram a importancia dos estudos relacionados às características psicológicas ao propor racionalidade e racionalidade limitada como um tema de tomada de decisão. Especificamente, sugere-se estudar como a cognição individual pode afetar a motivação do indi- viduo em engajar em conflito ou consenso na tomada de decisão estratégica.

Como sugestão futura propõe-se também estender os estudos sobre ética na tomada de decisão. Esses estudos estão relacionados às características perceptíveis ou psicológicas e a como elas podem afetar a tomada de decisão ética na organização, predominando os estudos sobre características perceptíveis.

Outro tema ainda pouco estudado na tomada de decisão é a religião. Esses estudos referem-se, geralmente, à religiosidade como característica perceptível do indivíduo, medindo como tal fator afeta a tomada de decisão. Sugere-se um aprofundamento do tema em pesquisas futuras.

\section{Conclusões e Sugestões}

Este artigo teve como objetivo compreender a estrutura intelectual que conecta teorias e autores que pesquisam a tomada de decisão estratégica do alto escalão e propor uma agenda para pesquisa futura em tomada de decisão estratégica. Para esse fim, utilizou-se de pesquisa bibliométrica de artigos publicados em periódicos internacionais, obtendo um total final de 277 artigos.

O tema tomada de decisão estratégica está dividido em três clusters teóricos. O cluster \#1 aborda trabalhos sobre características do TMT e a relação destas com o desempenho. Esse cluster foi divido em dois subclusters, características perceptíveis e características psicológicas. O autor mais citado é Donald Hambrick, seu trabalho mais referênciado no cluster e em toda amostra (ver Hambrick e Mason (1984)). Este e seus outros trabalhos sobre Alto Escalão servem como base para vários outros estudos do cluster. O cluster \#2 apresenta trabalhos sobre o ambiente em que são tomadas as decisões, juntamente com modelos e processos sobre tomada de decisão. Esse cluster apresenta dois subclusters, um com os trabalhos sobre o ambiente, o outro sobre modelos e processos decisórios. Kathleen Eisenhardt é a autora mais prolífica do cluster \#2. No terceiro cluster estão representados os trabalhos sobre como o conflito pode afetar o processo de tomada de decisão. O autor mais representativo desse cluster é Allen Amason, seu trabalho de 1996 serve como referencial para estudos sobre conflito. 
Outra contribuição deste trabalho foi a criação de uma agenda de pesquisas futura. Essa agenda tem como objetivo auxiliar a direcionar os esforços das pesquisas sobre tomada de decisão para temas úteis, pouco explorados e/ou com grande importância. Dentre os temas identificados ressalta-se a necessidade de se investigar características psicológicas do TMT, estudar como a religião, a ética e a emoção afetam o indivíduo, fazer estudos comparativos sobre como culturas afetam o processo de tomada de decisão $e$ integrar a outros temas.

Este artigo apresenta um conjunto de limitações. Primeiro, há limitações que são específicas aos estudos bibliométricos. Na medida em que assentam sobre análises estatísticas e contagens de citações $e$ cocitações, não incorporam análises qualitativas e de conteúdo efetivas dos trabalhos. Os estudos bibliométricos também utilizam fontes documentais escritas, estando dependetes dos periódicos disponíveis para amostragem. Ainda assim há a vantagem de se evitar ou de se reduzir eventuais vieses que possam existir na seleção dos artigos. Por se tratar de uma pesquisa bibliométrica, com o uso de uma base de dados - ISI Web Of Science, os resultados aqui obtidos não devem ser amplamente generalizados.

Outra limitação específica deste estudo é a interpretação dos dados encontrados, que é dependente do julgamento do autor, sendo que ele, por mais imparcial e literato no assunto de pesquisa, tem limitações humanas, sendo racionalmente limitado acerca das interpretações efetuadas no estudo (SIMON, 1991). As relações estabelecidas neste estudo não são fruto perfeito da literatura, já que diferentes ênfases, oriundas da interpretação do autor, podem gerar conclusões diferentes sobre o tema. Autores podem utilizar de uma mesma referência de formas diferentes para construir argumentos distintos. Logo, tem-se como limitação a validade do constructo elaborado.

\section{REFERÊNCIAS}

AMIT, R.; SCHOEMAKER, P. J. H. Strategic assets and organizational rent. Strategic Management Journal, USA, v. 14, n. 1, p. 33-46, 1993.

BARNEY, J. B. Firm resources and competitive advantage. Journal of Management, USA, v. 17, p. 99-120, 1991.

CAPES. 2010. Sistema WebQualis. [2010].

Disponível em: <http://qualis.capes.gov.

br/webqualis/publico/documentosDeArea.

seam?conversationPropagation $=$ begin $>$. Acesso em: $1^{\circ}$ mar. 2013.

CANNELLA JR., A. A. Upper echelons: Donald Hambrick on executives and strategy. Academy of Management Executive, Texas, v. 15, n. 3, p. 37-42, 2001.

COHEN, M. D.; MARCH, J. G.; OLSEN, J. P. A garbage can model of organizational choice. Administrative

Science Quarterly, USA, v. 17, p. 1-25, 1972.

COLLINS, J. Good to Great: why some companies make the leap and others don't. New York: HarperCollins, 2001.

DAS, T. K.; TENG, B. Cognitive biases and strategic decision processes: An integrative perspective. Journal of Management Studies, USA, v. 36, p. 757-778, 1999.

EISENHARDT, Kathleen K. Making Fast Strategic Decisions in High-Velocity Environments. The Academy of Management Journal, USA, v. 32, n. 3, 1989, p. 543-576.

EISENHARDT, K. K.; ZBARACKI, M. J. Strategic decisionmaking. Strategic Management Journal, USA, v. 13, p. 17-37, 1992.

ELBANNA, S. Strategic decision making: process perspectives. International Journal of Management Reviews, USA, v. 8, n .1, p. 1-20, 2006.

FERREIRA, M. et al. Entrepreneurship research: a bibliometric study of the EnANPADs 1997-2008.

REBRAE - Revista Brasileira de Estratégia, USA, v. 3, n. 1, p. 31-47, 2010. 
FINKELSTEIN, S.; HAMBRICK, D C. Chief Executive compensation: a synthesis and reconciliation. Strategic Management Journal, USA, v. 9, n. 6, p. 543-558, 1988.

FREDRICKSON, J. W. The comprehensiveness of strategic decision processes: extension, observations, future directions. Academic of Management Journal, USA, v. 27, n. 3, p. 445-466, 1984.

FREDRICKSON, J. W.; HAMBRICK, D. C.; BAUMRIN, S. A model of CEO dismissal. Academy of Management Review, New York, v. 13, n. 2, p. 255-270, 1988.

GARTNER, W. B. Who is an entrepreneur? Is the wrong question. American Journal of Small Business, USA, v. 12, n. 4, p. 11-32, 1988.

GRANT, R. On "dominant logic" and the link between diversity and performance. Strategic Management Journal, USA, v. 9, p. 41-58, 1988.

GUILERA, G.; BARRIOS, M.; GÓMEZ-BENITO, J. Meta-analysis in psychology: a bibliometric study, Scientometrics, USA, v. 94, p. 3, p. 943-954, 2013.

HAMBRICK, D. C. Environment, strategy and power within top management teams. Administrative Science Quarterly, New York, v. 26, n. 2, p. 253-276, 1981.

HAMBRICK, D. C.; MASON, P. A. Upper Echelons: the organization as a reflection of its top managers. Academy of Management Review, New York, v. 9, n. 2, p. 193206, 1984.

HAMBRICK, D. C.; FINKELSTEIN, S. Managerial discretion: A bridge between polar views of organizational outcomes. In: CUMMINGS, L. L.; STAW, B. M. (Ed.)

Research in Organizational Behaviour, Greenwich: JAI Press, v. 9, p. 369-406, 1987.

HAMBRICK, D. C.; FUKUTOMI, G. The seasons of a CEO's tenure. Academy of Management Review, New York, v. 16, n. 4, p. 719-742, 1991.

HAMBRICK, D. C.; D'AVENI, R. A. Top Management Team deterioration as part of the downward spiral of large bankruptcies. Management Science, Hanover Maryland, v. 38, n. 10, p. 1.445-1.466, 1992.
HAMBRICK, D. C. Fragmentation and the other problems CEOs have with their Top Management Teams. California Management Review, California, v. 37, n. 3, p. 110-127, 1995.

HAMBRICK, D. C.; CHO, T. S.; CHEN, M. J. The influence of Top Management Team heterogeneity on firms' competitive moves. Administrative Science Quarterly, New York, v. 41, n. 4, p. 659-684, 1996.

HAMEL, G.; PRAHALAD, C. K. Competing for the Future. Boston: Harvard Business School Press, 1994.

HASELTON, M. G.; NETTLE, D.; ANDREWS, P. W. The evolution of cognitive bias. In: BUSS, D. M. (Ed.). Handbook of Evolutionary Psychology. Hoboken, NJ: Wiley, 2005. p. 724-746.

HAYWARD, M. L. A.; HAMBRICK, D. C. Explaining the premiums paid for large acquisitions: evidence of $\mathrm{CEO}$ hubris. Administrative Science Quarterly, New York, v. 42, n. 1, p. 103-127, 1997.

HENDERSON, A.; MILLER, D.; HAMBRICK, D.C. How quickly do CEOs become obsolete? industry dynamism, CEO tenure and company performance. Strategic Management Journal, USA, v. 27, n. 5, p. 447-460, 2006.

HILLER, N.; HAMBRICK, D.C. Conceptualizing executive hubris: the role of (hyper-) core self-evaluations in strategic decision-making. Strategic Management Journal, USA, v. 26, n. 4, p. 297-319, 2005.

HOFFMAN, D.; HOLBROOK, M. The intellectual structure of consumer research: A bibliometric study of author cocitations in the first 15 years of the Journal of Consumer Research. Journal of Consumer Research, USA, v. 19, p. 505-517, 1993.

HOSKISSON, R. et al. Theory and research in strategic management: swings of a pendulum. Journal of Management, USA, v. 25, n. 3, p. 417-456, 1999.

KEENEY, Ralph L. Making Better Decision Makers. Decision Analysis, USA, v. 1, n. 4, p. 193-204, December, 2004, 
KOCHER, M. G.; SUTTER, M. Time is money - Time pressure, incentives, and the quality of decision-making. Journal of Economic Behavior \& Organisation, USA, v. 61, p. 375-392, 2006.

KUNC, M. H.; MORECROT, J. D. W. Managerial decision making and firm performance under a resource-based paradigm. Strategic Management Journal, USA, v. 31, p. 1.164-1.182, 2010.

LEWIN, A. Y.; STEPHENS, C. U. CEO attitudes as determinants of organization design: an integrated model. Organization Studies, USA, v. 15, p. 183-212, 1994.

MEDEIROS JR., R.; SERRA, F; FERREIRA, M. P. Alta administração como recurso estratégico: agenda de pesquisa brasileira a partir de estudo bibliográfico dos trabalhos de Hambrick. Revista Ibero-Americana de Estratégia, São Paulo, v. 8, n. 1, p. 46-65, 2009.

MILLER, D.; TOULOUSE, J. M. Chief executive personality and corporate strategy and structure in small firms. Management Science, USA, v. 32, n. 11, p. 1.389-1.409, 1986

MISCHEN, P. A.; JACKSON, S. K. Applying complexity theory, knowledge management and social network analysis to policy implementation. Public

Administration Quarterly, USA, v. 32, p. 314-338, 2008.

MORAN, M. et al. Alianças Estratégicas: uma análise bibliométrica da produção científica entre 1989 e 2008.

Revista de Ciências da Administração, Florianópolis, v. 12, n. 27, p. 42-62, 2010.

PAPADAKIS, V. M.; BARWISE, P. How much do CEOs and Top Managers Matter in strategic decision-making? British Journal of Management, USA, v. 13, p. 83-95, 2002.

PETERAF, M. The cornerstones of competitive advantage: A resource-based view. Strategic Management Journal, USA, v. 14, v. 3, p. 179-191, 1993.

PILKINGTON, A.; MEREDITH, J. The evolution of the intellectual structure of operations management 1980-2006: A citation/co-citation analysis. Journal of Operations Management, USA, v. 27, p. 185-202, 2009.
PORTER, M. E. Competitive strategy: techniques for analysing industry and competitors. New York: Free Press, 1980.

PORTER, M. E. Towards a Dynamic Theory of Strategy. Strategic Management Journal, USA, v. 12, p. 95117, 1991.

PRAHALAD, C. K.; BETTY, R. The dominant logic: a new linkage between diversity and performance. Strategic Management Journal, USA, v. 7, p. 485-501, 1986.

RAHMAN, N.; DE FEIS, G. L. Strategic decision-making models and methods in the face of complexity and time pressure. Journal of General Management, USA, v. 35. n. 2, p. 43-60, 2009.

RAJAGOPALAN, N.; RASHEED, A. M.; DATTA, D. K. Strategic Decision Processes: Critical Review and Future Directions. Journal of Management, USA, v. 19, n. 2, p. 349-384, 1993

RAMOS-RODRIGUEZ, A. R.; RUIZ-NAVARRO,

$\mathrm{J}$. Changes in the intellectual structure of strategic management research: a bibliometric study of the Strategic Management Journal, 1980- 2000. Strategic

Management Journal, USA, v. 25, p. 981-1.004, 2004.

RUMELT, R. P. Towards a strategic theory of the firm. In: LAMB, R. B. (Ed.). Competitive Strategic Management. Upper Sadler River: Prentice Hall, 1984. p. 95-117.

SANDERS, W. G.; HAMBRICK, D. C. Swinging for the fences: the effects of CEO stock options on company risk-taking and performance. Academy of Management Journal, New York, v. 50, n. 5, p. 1.055-1.078, 2007.

SCHWENK, C. R. Information, cognitive biases, and commitment to a course of action. Academy of Management Review, USA, v. 11, p. 298-310, 1986

SERRA, F; FERREIRA, M. P. Emerging determinants of firm performance: A case study research examining the strategy pillars from a resource-based view. Management Research: The Journal of the Iberoamerican Academy of Management, USA, v. 8, n. 1, p. 7-24, 2010. 
SERRA, F; ALMEIDA, M. R.; FERREIRA, M. P.

Organizational decline: a yet largely neglected topic in organizational studies. [2012]. Disponível em: <http:// globadvantage.ipleiria.pt/files/2012/02/working_paper-88_ globadvantage.pdf $>$. Acesso em: $1^{\circ}$ out. 2012.

SIGGELKOW, N.; RIVKIN, J. W. Designing organisations for turbulence and complexity. Organisation Science, USA, v. 16, p. 101-122, 2005.

SIMON, H. A. Rational decision-making in business organizations. American Economic Review, USA, v. 69, p. 493-513, 1979.

SIMON, H. Bounded Rationality and Organizational Learning. Organization Science, USA, v. 2, p. 125134, 1991.

\section{SELZNICK, P. Leadership in administration: a}

sociological interpretation. Evanston, IL: Row Peterson, 1957.

SMITH, K. G. et al. Top Management Team Demography and Process: the role of social integration and communication. Administrative Science Quarterly, New York, v. 39, n. 3, p. 412-438, 1994.

\section{THOMSON REUTERS, Web of Science Factsheet.}

2012. Disponível em: <http://thomsonreuters.com/ content/science/pdf/Web_of_Science_factsheet.pdf $>$. Acesso em: $1^{\circ}$ out. 2012.

VAARA, E.; WHITTINGTON, R. Strategy as Practice: taking social practices seriously. Academy of

Management Annals, USA, v. 6, n. 1, p. 285-336,

2012. 